\title{
Factors Associated with Mortality Among Patients with Central Venous Catheter- Related Bloodstream Infection in an Intensive Care Unit
}

\author{
${ }^{1}$ Priscilla Roberta Silva Rocha, ${ }^{2}$ Marcelo de Oliveira Maia, ${ }^{2}$ Gisele Brocco Magnan, \\ ${ }^{3}$ Juliano Carregaro, ${ }^{1,4}$ Francisco de Assis Rocha Neves and ${ }^{1,4}$ Angelica Amorim Amato \\ ${ }^{1}$ Postgraduate Program in Health Sciences, Faculty of Health Sciences, University of Brasilia, Brasilia, Brazil \\ ${ }^{2}$ Hospital Santa Luzia, Brasilia, Brazil \\ ${ }^{3}$ Department of Biology, Anhanguera Faculty, Brasilia, Brazil \\ ${ }^{4}$ Department of Pharmaceutical Sciences, Faculty of Health Sciences, University of Brasilia, Brazil
}

Received 2012-08-19, Revised 2012-11-30; Accepted 2012-11-30

\begin{abstract}
Central venous catheterization is a common practice in the management of critically ill patients and is associated with various complications, such as Bloodstream Infections (BSI), which are major determinants of increased morbidity, mortality and healthcare expenses. Few studies have addressed factors that predict mortality in patients with this complication. The aim of this study was to investigate factors associated with mortality in patients with Central Venous Catheter (CVC)-related BSI in an intensive care unit of a tertiary care hospital in the Federal District, Brazil. This was a retrospective and observational study, in which all CVC-related BSI that occurred between January 2008 and December 2010 were reviewed. We obtained demographic, clinical, biochemical and microbiological data from medical records and investigated its association with mortality during ICU stay. There were 4,504 ICU admissions during the study period and 68 were complicated by CVC-related BSI (4.09 per 1000 catheter-days), most due to gram-negative organisms (45.6\%). Overall mortality was 59.7\%. Death risk was significantly associated with mechanical ventilation (OR 27.8, 95\% CI 3.28-250, p<0.001), the mean number of invasive devices other than the CVC $(1.44$ Vs 2.37 in controls Vs cases, $p<0.001)$ and increased serum levels of urea (mean value of $44.2 \mathrm{mg} \mathrm{dL}^{-1}$ in survivors vs. $73.9 \mathrm{mg} \mathrm{dL}^{-1}$ in nonsurvivors, $\mathrm{p}=0.001$ ). Mortality was not associated with other clinical or biochemical features, neither with microbiological variables, although lethality was high among patients with gram-positive infections (77\% Vs $58.33 \%$ for fungi and $54.83 \%$ for gram-negative). CVC-related BSI was associated with high absolute mortality, which was predicted by mechanical ventilation and a higher number of invasive devices other than the CVC. Knowledge of local factors predictive of mortality is critical for planning strategies to reduce death risk associated with this complication.
\end{abstract}

Key words: Bloodstream Infection, Central Venous Catheter, Intensive Care Unit, Mortality

\section{INTRODUCTION}

Central venous catheterization is a common practice with unquestionable benefits in the management of critically ill patients (Mcgee and Gould, 2003). However, it is also associated with potentially serious complications (McGee and Gould, 2003;
Garnacho-Montero et al., 2008). One of the main complications is Central Venous Catheter (CVC)related Bloodstream Infection (BSI) (O'Grady et al., 2011), which is a major determinant of increased morbidity, mortality, hospitalization length and health care expenses in the Intensive Care Unit (ICU) (Pittet et al., 1994; Garrouste-Orgeas et al., 2006).

\section{Corresponding Author: Priscilla Roberta Silva Rocha, Postgraduate Program in Health Sciences, Faculty of Health Sciences,} University of Brasilia, Brasilia, Brazil 
In the United States, 80,000 new cases of CVCrelated BSI are estimated to occur in ICUs each year (Mermel et al., 2009). In South America, there are fewer studies addressing this issue. In an Argentinian 5 year study involving 6 hospitals, it was reported that CVCrelated BSI complicated $2.92 \%$ of all ICU admissions, increased hospitalization length by 11.9 days and resulted in a $24.6 \%$ excess mortality (Rosenthal et al., 2003). A Brazilian study carried out over a period of 11 months in 7 public ICUs in the Federal District found a $6.4 \%$ frequency of overall BSI and a $1.5 \%$ frequency of CVC-related BSI. This complication increased 3.5 times the duration of hospitalization (Mesiano and Merchan-Haman, 2007).

There are a number of interventions to attempt reducing the incidence of CVC-related BSI, mostly involving $\mathrm{CVC}$ insertion and care, such as barrier precautions, hand hygiene, skin antisepsis and careful CVC insertion site choice (O'Grady et al., 2002). Nevertheless, rationale and effective interventions to reduce CVC-related BSI rates and its associated mortality remain a major focus of research (Vincent et al., 2009) and identifying the factors that predict the occurrence of these infections and its associated mortality might contribute to improvement of these interventions. The aim of this study was to investigate factors associated with mortality in patients with central venous catheter CVCrelated BSI in an intensive care unit of a tertiary care hospital in the Federal District, Brazil.

\section{MATERIALS AND METHODS}

\subsection{Study Design}

This study was carried out in a 40-bed medicalsurgical ICU of a tertiary care hospital in Brasília, (Federal District, Brazil). All occurrences of CVCrelated BSI from January 2008 to December 2010 were prospectively investigated. The diagnosis of CVC-related BSI was defined according to the local protocol, which follows the recommendations from the Centers for Disease Control and Prevention, CDC (O'Grady et al., 2002; Mermel et al., 2009).

Patients aged over 18 years were included in the study and only the first episode of CVC-related BSI was considered if there was more than one episode of infection per patient during ICU stay. Exclusion criteria were CVC insertion prior to ICU admission, pregnancy and patients who were transferred to another ICU.

The following data were collected: age, gender, admission APACHE II score, reason for ICU admission, comorbidities, need for mechanical ventilation and renal replacement therapy, need for glucocorticoid or insulin therapy during ICU stay, need for other invasive devices, length of ICU stay and time period between admission and CVC-related BSI diagnosis. Biochemical (serum urea, creatinine, glucose and lactate levels), CVC-related (insertion site) and microbiological (microbiological isolate causing the infection: gram-positive, gram-negative or fungi) data were collected from medical records.

The study was approved by the local ethics committee, which required no written informed consent because it was an observational and retrospective study based on routinely obtained information in the ICU.

\subsection{Statistical Analysis}

Continuous variables were reported as the appropriate measures of central tendency and categorical variables, as percentages. CVC-related BSI rate was reported as incidence density (number of case per 1000 catheterdays). The patient status (survivor or non-survivor) at the end of ICU stay was considered as the outcome variable. The association of the outcome variable with the categorical variables analyzed was expressed as the Odds Ratio (OR) with $95 \%$ Confidence Intervals $(95 \% \mathrm{CI})$. The frequency of these variables in survivors and nonsurvivors groups was compared using simple logistic regression. For continuous variables showing normal distribution, mean values were compared using t-Student test. For those not showing normal distribution, median values were compared using Mann-Whitney and Kruskal-Wallis tests. Statistical significance was set at pvalue $<0.05$. Statistical analyses were done using the 17th version of the Statistical Package for the Social Sciences (Chicago, IL, US) and R Statistics softwares.

\section{RESULTS}

\subsection{Study Population}

A total of 4,504 patients were admitted to the ICU during the study period and CVC-related BSI was diagnosed in 68 patients $(1.53 \%)$. The corresponding incidence density of this infection was 4.09 per 1000 catheter-days. All cases were included in the study.

Patients with CVC-related BSI had a mean age of $72.20 \pm 17.0$ years and $54.4 \%$ were male. Mean APACHE II score on admission was $16.4 \pm 5.6$ and the mean duration of ICU stay was $91.22 \pm 77.40$ days. The most common reason for ICU admission was medical conditions associated with infection $(50.0 \%)$ and the most frequent comorbidity was diabetes $(27.9 \%)$, as shown in Table 1.

The most commonly needed invasive procedure was mechanical ventilation $(82.1 \%)$ and $22.4 \%$ of patients needed hemodialysis as renal replacement therapy. 
Table 1. Demographic and clinical features of patients with CVC-related BSI

\begin{tabular}{lc}
\hline & Total study period (2008-2010) N $=68$ \\
\hline Demographic data & $72.20 \pm 17.0$ \\
Age (mean \pm SD) & 54.4 \\
Male gender (\%) & $16.4 \pm 5.6$ \\
Clinical data & $91.22 \pm 77.40$ \\
APACHE II at admission (mean \pm SD) & \\
Length of ICU stay in days (mean \pm SD) & 16.6 \\
ICU admission reason & 50.0 \\
Clinical without infection (\%) & 22.1 \\
Clinical with infection (\%) & 11.8 \\
Surgical without infection (\%) & 27.9 \\
Surgical with infection (\%) & 10.3 \\
Comorbidities & 10.3 \\
Diabetes mellitus (\%) & 82.1 \\
Oncologic disease (\%) & 22.4 \\
Chronic renal disease (\%) & 2.0 \\
Need for mechanical ventilation (\%) & $38.2 \pm 28.8$ \\
Need for dialysis (\%) & \\
Mean number of invasive devices other than the CVC & 94.0 \\
Time period between ICU admission and diagnosis of & 31.3 \\
CVC-related BSI (mean \pm SD) & \\
Insulin therapy (\%) & \\
Glucocorticoid therapy (\%) & \\
CVC insertion site & 7.5 \\
Subclavian vein (\%) & \\
Internal jugular vein (\%) & 1.5 \\
Femoral vein (\%) &
\end{tabular}

Table 2. Demographic and clinical features and death risk among patients with CVC-related BSI

\begin{tabular}{|c|c|c|c|c|}
\hline & Survivors $N=27$ & Non-survivors $\mathrm{N}=41$ & OR $(95 \%$ IC) & p-value \\
\hline Mean age \pm SD & $69.3 \pm 15.0$ & $74.1 \pm 18.3$ & & 0.080 \\
\hline Male gender & $13(48.14 \%)$ & $24(58.3 \%)$ & $1.52(0.57-4.04)$ & 0.277 \\
\hline Apache II at admission (mean $\pm \mathrm{SD}$ ) & $15.7 \pm 4.9$ & $16.9 \pm 6.0$ & & 0.700 \\
\hline \multicolumn{5}{|l|}{ ICU admission reason } \\
\hline Clinical without infection & $4(14.8 \%)$ & $7(17.1 \%)$ & $0.84(0.22-3.2)$ & 0.542 \\
\hline Clinical with infection & $11(40.7 \%)$ & $23(56.1 \%)$ & $1.86(0.69-4.9)$ & 0.161 \\
\hline Surgical without infection & $7(25.9 \%)$ & $8(19.5 \%)$ & $0.69(0.22-2.2)$ & 0.369 \\
\hline Surgical with infection & $5(18.5 \%)$ & $3(7.3 \%)$ & $2.9(0.63-13.2)$ & 0.154 \\
\hline \multicolumn{5}{|l|}{ Comorbidities } \\
\hline Oncologic disease & $1(3.7 \%)$ & $6(14.6 \%)$ & $4.46(0.50-40.0)$ & 0.148 \\
\hline Diabetes mellitus & $7(25.9 \%)$ & $12(29.3 \%)$ & $1.18(0.4-3.52)$ & 0.494 \\
\hline Chronic renal disease & $4(14.8 \%)$ & $3(7.3 \%)$ & $0.45(0.09-2.2)$ & 0.275 \\
\hline Need for mechanical ventilation & $16(59.3 \%)$ & $40(97.6 \%)$ & $27.8(3.28-250)$ & $<0,001$ \\
\hline Need for dialysis & $6(22.2 \%)$ & $9(22 \%)$ & $1.02(0.31-3.3)$ & 0.603 \\
\hline Glucocorticoid therapy & $8(29.6 \%)$ & $13(31.7 \%)$ & $1.10(0.38-3.2)$ & 0.537 \\
\hline Insulin therapy & $26(96.3 \%)$ & $38(92.7 \%)$ & $0.49(0.05-4.9)$ & 0.478 \\
\hline \multicolumn{5}{|l|}{ CVC insertion site } \\
\hline Subclavian vein & $25(92.6 \%)$ & $37(90.2 \%)$ & $1.35(0.23-7.9)$ & 0.551 \\
\hline Internal jugular vein & $1(3.7 \%)$ & $4(9.8 \%)$ & $2.8(0.29-26.3)$ & 0.334 \\
\hline Femoral vein & $1(3,7 \%)$ & A0 & - & - \\
\hline $\begin{array}{l}\text { Mean number of invasive } \\
\text { devices other than the CVC }\end{array}$ & $1.44 \pm 1.0$ & $2.37 \pm 0.8$ & & $<0.001$ \\
\hline Mean length of ICU stay in days \pm SD & $87.6 \pm 6.8$ & $93.6 \pm 78.7$ & & 0.878 \\
\hline
\end{tabular}

CVC: Central Venous Catheter; ICU: Intensive Care Unit; SD: Standard Deviation; OR: Odds Ratio, 95\% IC: 95\% confidence interval 
Table 3. Biochemical features and death risk among patients with CVC-related BSI

\begin{tabular}{llll}
\hline & Survivors $\mathrm{N}=27$ & Non-survivors $\mathrm{N}=41$ & $\mathrm{p}$-value \\
\hline Urea, $\mathrm{mg} / \mathrm{dL}($ mean $\pm \mathrm{SD})$ & $44.2 \pm 42.1$ & $73.9 \pm 45.0$ & 0.001 \\
Creatinine, $\mathrm{mg} / \mathrm{dL}(\mathrm{mean} \pm \mathrm{SD})$ & $1.2 \pm 1.3$ & $1.4 \pm 1.5$ & 0.411 \\
Glucose, $\mathrm{mg} / \mathrm{dL}($ mean $\pm \mathrm{SD})$ & $123 \pm 34.6$ & $148.2 \pm 70.2$ & 0.125 \\
Lactate, $\mathrm{mmol} / \mathrm{L}($ mean $\pm \mathrm{SD})$ & $1.2 \pm 0.97$ & $2.1 \pm 2.7$ & 0.093 \\
\hline
\end{tabular}

SD: Standard Deviation

Table 4. Microbiological diagnosis and death risk among patients with CVC-related BSI

\begin{tabular}{llllll}
\hline & $\mathrm{N}(\%)$ & Mortality N (\%) & Lethality (\%) & OR (95\%IC) & p-value \\
\hline Gram-positive & $13(19.1)$ & $10(14.7)$ & 77.00 & $2.6(0.64-10.4)$ & 0.147 \\
Gram-negative & $31(45.6)$ & $17(25)$ & 54.83 & $0.65(0.23-1.7)$ & 0.277 \\
Fungi & $24(35.3)$ & $14(20.58)$ & 58.33 & $0.88(0.32-2.4)$ & 0.504 \\
\hline
\end{tabular}

OR: Odds Ratio, $95 \%$ IC: $95 \%$ confidence interval

The mean number of invasive devices other than the CVC was 2.0 and the mean time period between ICU admission and diagnosis of CVC-related BSI was $38.2 \pm 28.8$ days. The need for insulin (94\%) and glucocorticoid $(31.3 \%)$ therapy was frequent. The most common CVC insertion site was the subclavian vein (Table 1). Biochemical data collected within a 5 day period of CVC-related BSI diagnosis showed mean serum levels of urea of $62.1 \pm 46.5 \mathrm{mg} \mathrm{dL}^{-1}$ (reference range, RR 10-46), creatinine of $1.3 \pm 1.4 \mathrm{mg} \mathrm{dL}^{-1}$ (RR $0.6-1.1)$, glucose of $138.2 \pm 59.7 \mathrm{mg} \mathrm{dL}^{-1}$ and lactate of $1.7 \pm 2.3 \mathrm{mmol} / \mathrm{L}$ (RR 0.4-2.0). Most cases of CVCrelated BSI were due to gram-negative organisms $(45.6 \%)$, followed by fungi $(35.3 \%)$ and gram-positive (19.1\%) organisms. There were no cases of polymicrobial CVC-related BSI.

\subsection{Factors Associated with Mortality among Patients with CVC-Related BSI}

In the study period, there were 41 deaths among patients with CVC-related BSI, corresponding to a mortality of $59.7 \%$. There was a significant association between death risk and the need for mechanical ventilation (OR 27.8, IC $95 \% 3.28-250.00, \mathrm{p}<0.001)$ and the mean number of invasive devices other than the CVC (1.44 Vs 2.37 in the survivors vs. non-survivors group, $\mathrm{p}<0.000)$. There was a trend towards association between mortality and the presence of oncologic disease (OR 4.46), although not statistically significant (IC 95\% 0.50-40.0, $\mathrm{p}=0.148$ ). There was no association between death risk and the other demographic and clinical variables analyzed such as admission APACHE II score, diabetes, chronic renal disease, the need for hemodialysis and the mean length of ICU (Table 2).

Interestingly, serum levels of urea, but not those of creatinine, were significantly associated with death risk (mean urea serum levels of $44.2 \mathrm{mg} \mathrm{dL}^{-1}$ in survivors Vs $73.9 \mathrm{mg} \mathrm{dL}^{-1}$ in non-survivors, $\mathrm{p}=0.001$ ). Other biochemical variables were not associated with mortality, as shown in Table 3. Mortality was higher $(25.0 \%)$ in patients with CVC-related BSI due to gram-negative organisms, although patients with gram-positive infections showed the highest lethality (77\%). However, no association was found between microbiological diagnosis and death risk in patients with CVC-related BSI (Table 4).

\section{DISCUSSION}

Nosocomial BSIs are primarily associated with intravascular devices, such as CVCs and represent a major determinant of morbidity and mortality among critically ill patients (Laupland et al., 2002; Vincent, 2003; Garrouste-Orgeas et al., 2006; Vincent et al., 2009). We studied 4,504 ICU admissions over a period of three years; $1.53 \%$ of them were complicated by CVC-related BSI and the correspondent incidence density was 4.09 per 1000 catheter-days. Patients with CVC-related BSI in our study were old (mean age of 72.20 years) and there was a slight male preponderance $(54.4 \%)$. We found a high overall mortality in patients with this complication (59.7\%).

There is a large variation in the incidence and mortality rates of CVC-related BSI in the ICU setting worldwide. These reasons for these discrepancies are not completely defined, but are believed to reflect at least in part the diversity of local clinical practices, distinct patient characteristics and the different designs of epidemiological studies from which these data are derived (Tacconelli et al., 2009; O'Grady et al., 2011). Data from the SCOPE (Surveillance and Control of Pathogens of Epidemiologic Importance) study, 
comprising 49 hospitals in the United States, indicated an incidence of 60 cases of nosocomial BSI per 10,000 hospital admissions, $50.5 \%$ of which occurred in the ICU and a crude mortality of all BSI of $27 \%$ (Wisplinghoff et al., 2004). In France, the National Nosocomial Infection Survey estimated an incidence rate of CVC-related BSI of 1.23 per 1000 cathetersdays (Tacconelli et al., 2009) and another European study reported a $43.9 \%$ mortality of BSI in the ICU (Pratikaki et al., 2009). In South America, an Argentinian study found a $2.92 \%$ incidence of CVCrelated BSI in the ICU, with a crude mortality of $52.4 \%$ (Rosenthal et al., 2003). A Brazilian study also conducted in the ICU setting reported a $1.5 \%$ frequency of CVC-related BSI with a $40 \%$ overall mortality (Mesiano and Merchan-Haman, 2007). We found an incidence of CVC-related BSI similar to the latter study, although mortality in our study population was considerably higher. This finding might be attributable to some of the patient's features, which are predictive of increased overall mortality, such as advanced age, illness severity on ICU admission as indicated by a high APACHE II score (mean of 16.4), serious underlying diseases (malignancy, diabetes and chronic renal disease) and the common need for other invasive procedures (Garnacho-Montero et al., 2008; Stoeckle et al., 2008; Son et al., 2010).

In addition, in this study death risk was associated with the need for mechanical ventilation and with a higher mean number of invasive devices other than the CVC. It was also associated with urea but not creatinine serum levels and this may reflect the advanced age of our study population. No significant differences were found for other variables analyzed. In other studies, several factors have been described as predictive of mortality in patients with CVC-related BSI, such as adult respiratory distress syndrome, shock, multiorgan failure, mechanical ventilation, chronic hepatic failure, acute renal failure and APACHE II score greater than 14 (Rello et al., 1994; Valles et al., 1997; Garrouste-Orgeas et al., 2006). Unlike these studies, we could not find an association of mortality with admission APACHE II score. It is also noteworthy that factors associated with mortality risk, including those found in our study, may all be viewed as surrogate markers of illness severity, rather than specific etiologic factors for mortality.

We found a predominance of CVC-related BSI infections due to gram-negative organisms over fungi and gram-positive. Although this is in disagreement with the more recent reports indicating an increased incidence of gram-positive organisms in patients with BSI in the
ICU setting (Wisplinghoff et al., 2004), it may be explained by local factors and also by the increased age of our study population and the severity of their clinical status, which have been found to be associated with increased risk for gram-negative infections (Waterer and Wunderink, 2001; Kohlenberg et al., 2008; Vincent et al., 2009). Mortality was higher among patients with CVCrelated BSI due gram-negative bacteria (25\%), in agreement with data from the Extended Prevalence of Infection in Intensive Care (EPIC II) study, which comprised 75 countries and found that mortality was higher among ICU patients with infections due to gramnegative organisms (Vincent et al., 2009). Lethality, however, was higher among patients with infections due to gram-positive organisms $(77 \%)$ in our study.

This study has some limitations, including the relatively small absolute number of CVC-related BSI occurrences and the high absolute mortality in patients with this complication, which may have precluded us from finding differences between survivors and non-survivors. Moreover, our study did not include a control group of patients submitted to CVC insertion but without CVCrelated BSI and it was limited to only one ICU facility.

\section{CONCLUSION}

We found a high mortality in patients with CVCrelated BSI, which was associated with need for mechanical ventilation, with a higher mean number of invasive devices other than the CVC and with a higher serum level of urea. This suggests that in addition to rigorous infection control measures to reduce the incidence of this complication in the ICU setting, it is important to develop strategies to improve patient outcome when CVCrelated BSI infection occurs and these strategies should focus specially on severely ill patients.

\subsection{Competing Interests}

The authors declare that they have no competing interests. Authors' contributions: All authors contributed to the writing of the manuscript. All authors read and approved the final manuscript.

\section{REFERENCES}

Garnacho-Montero, J., T. Albado-Pallas, M. PalomarMartinez, J. Valles and B. Almirante et al., 2008. Risk factors and prognosis of catheter-related bloodstream infection in critically ill patients: A multicenter study. Intensive Care Med., 34: 2185 2193. DOI: $10.1007 / \mathrm{s} 00134-008-1204-7$ 
Garrouste-Orgeas, M., J.F. Timsit, M. Tafflet, B. Misset and J.R. Zahar et al., 2006. Excess risk of death from intensive care unit-acquired nosocomial bloodstream infections: A reappraisal. Clin. Infect. Dis., 42: 1118-1126. PMID: 1657572

Kohlenberg, A., F. Schwab, C. Geffers, M. Behnke and H. Rüden et al., 2008. Time-trends for Gramnegative and multidrug-resistant Gram-positive bacteria associated with nosocomial infections in German intensive care units between 2000 and 2005. Clin. Microbiol. Infect., 14: 93-96. PMID: 18034861

Laupland, K.B., D.A. Zygun, H.D. Davies, D.L. Church and T.J. Louie et al., 2002. Population-based assessment of intensive care unit-acquired bloodstream infections in adults: Incidence, risk factors and associated mortality rate. Crit. Care Med., 30: 2462-2467. PMID: 12441755

McGee, D.C. and M.K. Gould, 2003. Preventing complications of central venous catheterization. N. Engl. J. Med., 348: 1123-1133. DOI: 10.1056/NEJMra011883

Mermel, L.A., M. Allon, E. Bouza, D.E. Craven and P. Flynn et al., 2009. Clinical practice guidelines for the diagnosis and management of intravascular catheter-related infection: 2009 update by the Infectious Diseases Society of America. Clin. Infect. Dis., 49: 1-45. DOI: 10.1086/599376

Mesiano, E.R.A.B. and E. Merchan-Haman, 2007. Bloodstream infections among patients using central venous catheters in intensive care units. Rev. LatinoAm. Enfermagem, 15: 453-459. PMID: 17653429

O'Grady, N.P., M. Alexander, E.P. Dellinger, J.L. Gerberding and S.O. Heard et al., 2002. Guidelines for the prevention of intravascular catheter-related infections. Centers for Disease Control and Prevention. MMWR Recomm. Rep., 51: 1-29. PMID: 12233868

O'Grady, N.P., M. Alexander, L.A. Burns, P. Dellinger and J. Garland et al., 2011. Guidelines for the prevention of intravascular catheter-related infections. Clin. Infect. Dis., 52: e162-e193. DOI: 10.1093/cid/cir257

Pittet, D., D. Tarara and R.P. Wenzel, 1994. Nosocomial bloodstream infection in critically ill patients. Excess length of stay, extra costs and attributable mortality. JAMA, 271: 1598-1601. PMID: 8182812

Pratikaki, M., E. Platsouka, C. Sotiropoulou, C. Vassilakopoulos and O. Paniara et al., 2009. Risk factors for and influence of bloodstream infections on mortality: A 1-year prospective study in a Greek intensive-care unit. Epidemiol. Infect., 137: 727735. DOI: $10.1017 / \mathrm{S} 0950268808001271$
Rello, J., M. Ricart, B. Mirelis, E. Quintana and M. Gurgui et al., 1994. Nosocomial bacteremia in a medical-surgical intensive care unit: Epidemiologic characteristics and factors influencing mortality in 111 episodes. Intensive Care Med., 20: 94-98. PMID: 8201105

Rosenthal, V.D., S. Gusman, O. Migone and C.J. Crnich, 2003. The attributable cost, length of hospital stay and mortality of central line-associated bloodstream infection in intensive care departments in Argentina: A prospective, matched analysis. Am. J. Infect. Control, 31: 475-80. PMID: 14647110

Son, J.S., J.H. Song, K.S. Ko, J.S. Yeom and H.K. Ki et al., 2010. Bloodstream infections and clinical significance of healthcare-associated bacteremia: A multicenter surveillance study in Korean hospitals. J. Korean Med. Sci., 25: 992-998. DOI: 10.3346/jkms.2010.25.7.992

Stoeckle, M., C. Kaech, A. Trampuz and W. Zimmerli, 2008. The role of diabetes mellitus in patients with bloodstream infections. Swiss Med. Wkly., 138: 512-519. PMID: 18792825

Tacconelli, E., G. Smith, K. Hieke, A. Lafuma and P. Bastide, 2009. Epidemiology, medical outcomes and costs of catheter-related bloodstream infections in intensive care units of four European countries: Literature- and registry-based estimates. J. Hosp. Infect., 72: 97-103. DOI: 10.1016/j.jhin.2008.12.012

Valles, J., C. Leon, F. Alvarez-Lerma, 1997. Nosocomial bacteremia in critically ill patients: A multicenter study evaluating epidemiology and prognosis. Spanish Collaborative Group for Infections in Intensive Care Units of Sociedad Espanola de Medicina Intensiva y Unidades Coronarias (SEMIUC). Clin. Infect. Dis., 24: 387-395. PMID: 9114190

Vincent, J.L., 2003. Nosocomial infections in adult intensive-care units. Lancet, 361: 2068-2077. DOI: 10.1016/S0140-6736(03)13644-6

Vincent, J.L., J. Rello, J. Marshall, E. Silva and A. Anzueto et al., 2009. International study of the prevalence and outcomes of infection in intensive care units. JAMA, 302: 2323-2329. DOI: 10.1001/jama.2009.1754

Waterer, G.W. and R.G. Wunderink, 2001. Increasing threat of Gram-negative bacteria. Crit. Care Med., 29: N75-N81. PMID: 11292879

Wisplinghoff, H., T. Bischoff, S.M. Tallent, H. Seifert and R.P. Wenzel et al., 2004. Nosocomial bloodstream infections in US hospitals: Analysis of 24,179 cases from a prospective nationwide surveillance study. Clin. Infect. Dis., 39: 309-317. PMID: 15306996 